\title{
Characterizing Ice Contamination Using Leginon
}

Anchi Cheng, Denis Fellmann, Jim Pulokas, Joel Quispe, Francisco Guerra, Christian Sulloway, Clint Potter and Bridget Carragher

National Resource for Automated Molecular Microscopy and Department of Cell Biology, The Scripps Research Institute, La Jolla, CA 92037

Build up of contamination on TEM grids of samples preserved in vitreous ice is a common but seldom well characterized problem. A simple time-course measurement involves recording of the electron beam intensity on an area of the grid covered by a layer of ice (I) and calibrating this against the beam intensity measured at a clear area of the grid (I0). The optical density computed as $\ln (\mathrm{I} 0 / \mathrm{I})$ is then proportional to the ice thickness $[1,2]$. While these data are generally straight forward to obtain, it can be very tedious for an operator to complete a long systematic sereis of experiments. We have thus adapted our automated data acquisition system, Leginon [3], for this purpose and are currently acquiring and assessing a variety of ice thickness and contamination data.

All measurements were obtained using a Tecnai F20 transmission electron microscope equipped with a Gatan 4k CCD. As a test specimen we used a sample of TMV filaments preserved in vitreous ice suspended over Quantifoil grids (QUANTIFOIL Micro Tools GmbH, Germany). A Gatan 626 cryostage was used to transfer the specimen into the TEM. For a given grid the Leginon software is used to target a set of grid squares and on each square two holes are selected. One of the holes will be covered by ice in the normal range that we use for imaging and the other is cleared of ice by focusing the beam and burning it away. For each test square, an image is acquired at a magnification of $1100 x$, so that the entire square is viewable (see Figure 1a), and then two subsequent images are acquired of the targeted holes at a magnification of 5000x (see Figure 1b and c). At the same time the images are analyzed to measure the intensity values at the center of each targeted hole using both the "square" (1100x) and the "hole" images (5000x). These measurements are then systematically repeated at selected intervals for a specified period of time. All images, acquisition parameters and intensity values are stored to a relational database [4].

In a preliminary experiment we obtained measurements over a 13 hour time period. At the conclusion of the experiment the database was queried for the stored intensity measurements and these were converted to optical density and plotted as shown in Figure 2. The rate of change of optical density provides a measure of the rate of contamination of the surface of the grid. The observed contamination rate was constant, did not vary between different locations on the grid and was the same whether measured from the "square" or the "hole" images.

We plan to investigate other variables that are commonly thought to affect the contamination rate using similar controlled experiments. For example we will investigate the effects of the cold trap and whether the use of photographic film plates in the microscope has a deleterious effect on contamination. Further, we will test whether the rate of ice build up depends on the amount of radiation accumulated by the grid.

References

[1] Eusemann, R., et al., J. Microscopy 128(1982): 239-247. 
[2] Lepault, J., et al., J. Microscopy 129(1982): 89-102.

[3] Suloway, C., et al., J. Struct. Biol. (submitted).

[4] Fellmann, D., et al., J. Struct. Biol. 137(2002): 273-282.

[5] This work was conducted at the National Resource for Automated Molecular Microscopy which is supported by the NIH National Center for Research Resource (RR17573).
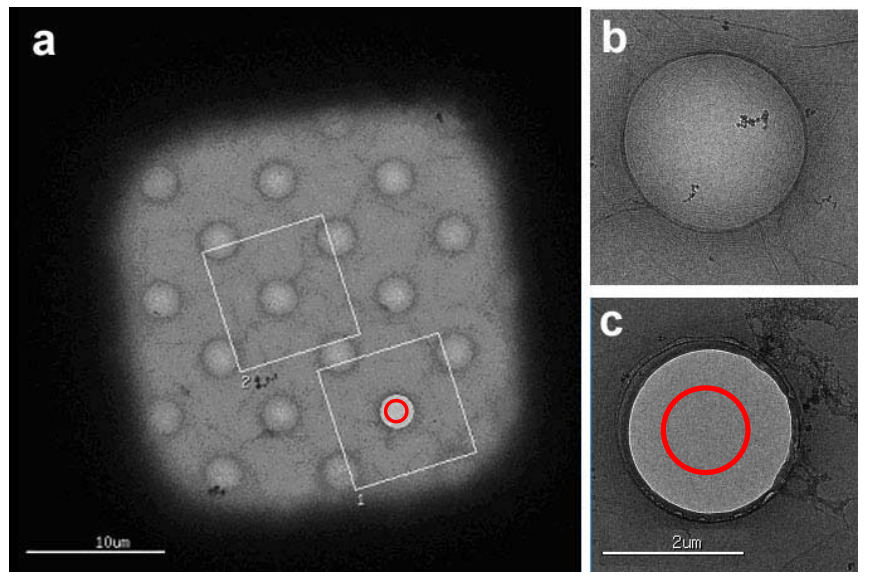

Figure 1: Configuration used in ice contamination measurement. (a) The 1100x image showing the entire square. Two holes were selected for higher magnification imaging as in (b) and (c). The red circles in (a) and (c) indicate the region of the hole where the mean intensity was calculated.

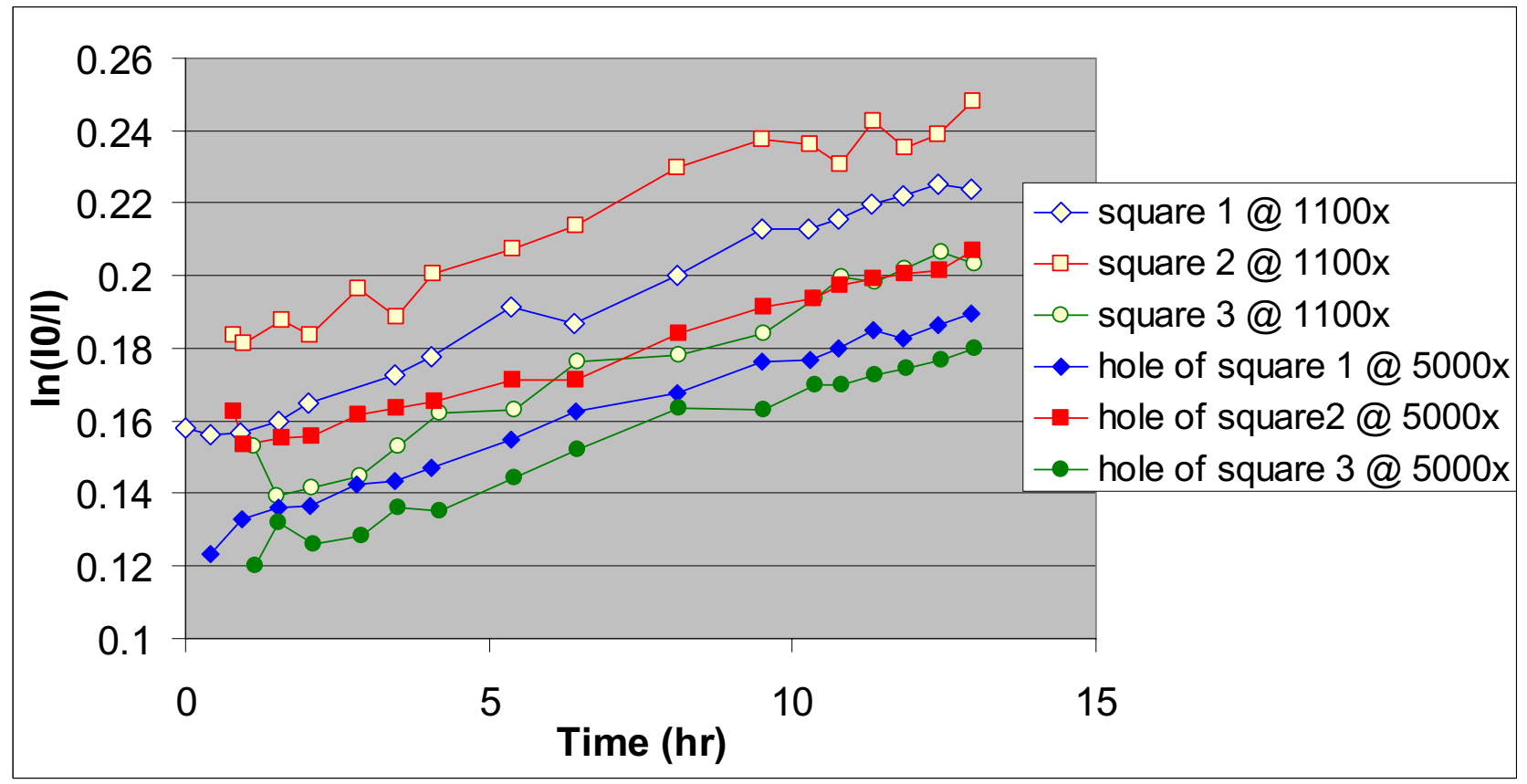

Figure 2: Increase of ice thickness over time measured from the optical density of $2 \mu \mathrm{m}$ holes covered by vitreous ice. I and I0 are measured from an ice filled hole and an empty hole, respectively, within the same grid square at the same time point. Measurements were made at 1100x and 5000x. The three grid squares used in this measurement were widely distributed across the grid. 